\title{
CONSECUENCIAS DEL MALTRATO GRAVE INTRAFAMILIAR EN LA INFANCIA: CONCEPTUALIZACIÓN Y DIAGNÓSTICO DE LAS REACCIONES POSTRAUMÁTICAS COMPLEJAS
} CONSEQUENCES OF SEVERE INTRAFAMILIAL MALTREATMENT DURING CHILDHOOD:
CONCEPTUALIZATION AND DIAGNOSIS OF COMPLEX POSTTRAUMATIC REACTIONS

\author{
López-Soler, C..'; Castro, M.'; Daset, L. R. ${ }^{2}$; Alcántara, M.'; \\ Fernández, V.'; Prieto, M. ${ }^{1}$ y Puerto, J. C. ${ }^{1}$ \\ 'Universidad de Murcia, España \\ 2Universidad Católica del Uruguay, Uruguay
}

\begin{abstract}
Resumen: El trauma psíquico es una transversalidad en la historia de la humanidad, pero de diagnóstico tardío, especialmente en lo que refiere a su incorporación a los sistemas de clasificación en psicopatología como Trastorno por Estrés Postraumático (TEPT). Desde ese momento hasta nuestros días han ido en aumento los estudios sobre TEPT, avanzando primero en las formas de evaluación específica y posteriormente en las propuestas de intervención, con énfasis en población adulta. Otros trabajos han evidenciado que, la semiológica del TEPT en niños y adolescentes, no puede ser analizada y abordada de igual manera que en población adulta. En este artículo se presentan los resultados generales de estudios sobre el TEPT en la infancia y adolescencia y los trabajos sobre un Listado de apoyo a los Clínicos para la evaluación del TEPT y del TEPTC O DESNOS, con algunos de los resultados preliminares de los estudios realizados con población de menores en situación de abuso y maltrato.
\end{abstract}

Palabras claves: TEPT; DESNOS; Reacciones postraumáticas; Trauma complejo; Maltrato infantil.

\begin{abstract}
Psychic trauma is a transversality in human history. Even though, its late diagnosed has been occurring, especially referring to its incorporation in psychopathological taxonomies as Postraumatic Stress Disorder (PSD). Since then, studies on PSD have been increased in quantity, firstly considering specific diagnostic issues, and secondly, tackling treatment proposals, generally with an adult population emphasis. Reports have shown that PSD clinical assessment can not be approached and analyzed in the same way in children and adolescents as it has been in adults. In this paper, general findings are presented about the use of a Supporting List for clinicians, designed to assess DESNOS. Preliminary results of a study on abuse and maltreatment situation are shown.
\end{abstract}

Key Words: PTSD; DESNOS; Posttraumatic reactions; Complex trauma; Childhood mis-

\section{CONCEPTUALIZACIÒN INICIAL DEL TEPT}

El trauma es una realidad que ha estado presente a lo largo de la historia de la humanidad (Cazabat, 2001), si bien el diagnóstico de Trastorno por Estrés Postraumático (TEPT) se incorpora formalmente en 1980 con su inclusión en la tercera edición del Manual Diagnóstico y
Estadístico de los Trastornos Mentales (DSM-III) (Valdivia, 2002), con el fin de recoger los efectos psicopatológicos de traumatización de los veteranos de Vietnam poco después del final de la guerra (van der Kolk, 2001). A partir de aquel momento, el interés en este trastorno ha ido creciendo exponencialmente (Cazabat, 2001) con una gran cantidad de estudios apoyando su validez etiológica y fenomenológica, como por

Correspondencia: Dra. Concepción López Soler, Profesora Titular de la Universidad de Murcia yVinculada Clínica Servicio Pediatría Hospital Universitario V. Arrixaca, Murcia. Correo Electrónico: clopezs@um.es 
ejemplo, los llevados a cabo por Atkinson, Sparr y Sheff en 1984; Hidalgo y Davidson en 2000; Mc-Farlane en 1988 o van Kampen, Watson y Tilleskjor en 1986.

Sin embargo, el TEPT en la población pediátrica ha sido poco investigado. Así, hasta 1987 no se reconoce en los criterios diagnósticos, que las reacciones de los niños/as al trauma pueden diferir de las de los adultos. Las altas tasas de exposición a sucesos traumáticos entre menores y el impacto psicológico de estas exposiciones, ha sido foco de atención sólo recientemente por parte de investigadores y clínicos en el ámbito de la salud mental. Aunque en los últimos años se han presentado estudios respecto al estrés postraumático en niños/as, aún es escasa la productividad científica sobre el tema, los pocos trabajos existentes carecen -en su mayoría- de rigor metodológico; las técnicas aplicadas no están adaptadas al nivel de desarrollo del menor y las muestras no son representativas.

EI TEPT es distinto de la mayoría de los trastornos psicológicos, al requerir para su diagnóstico la existencia de un estresor inicial. Los eventos traumáticos que pueden desencadenarlo, aunque no de forma exclusiva son: los desastres naturales e industriales, los combates al frente de la guerra, los ataques personales violentos, los secuestros, torturas, los accidentes de tráfico, el diagnóstico de enfermedades potencialmente mortales o los eventos traumáticos experimentados por otras personas que son transmitidos al individuo. En concreto, los sucesos más habitualmente relacionados con un TEPT en la infancia se refieren a exposición a violencia de distinto tipo: guerra, maltrato, accidentes, ser testigos de homicidios, suicidios, violaciones, ataques graves por animales, abuso físico o sexual, catástrofes humanas o naturales (Chiape, 2000; March, Amaya y Pynoos, 1997; Milgram, 1993).

Los menores pueden desarrollar TEPT a través de exposición directa, por observación y también a partir de la transmisión verbal de una información traumática. Si bien, como señalan Perrin, Smith y Yule (2000), no todas las personas que se han expuesto a un trauma desarrollarán TEPT. Debido a que solo aproximadamente una cuarta parte de quienes se exponen a un evento traumático desarrollan TEPT y a pesar de la alta prevalencia de la exposición a un suceso de este tipo, existirían otros factores asociados que aumentan el riesgo de desarrollar este trastorno (Palacios y Heinze, 2002).

Entre los factores predictores de respuesta traumática estudiados se encuentran: la naturaleza del evento traumático y el nivel de exposición a él; el género, la edad y el nivel de desarrollo; el nivel de funcionamiento premórbido -tanto del menor como de su familiay factores sociales (Gurwitch, Sullivan y Long, 1998; Pfefferbaum, 1997).

Con respecto a las características del hecho traumático tienen más posibilidades de desarroIlar un TEPT quienes estén expuestos a hechos traumáticos, como:

los infringidos por la obra del ser humano; por familiares directos o personas en las que se debía confiar; los que son repetidos y reiterados; mas aún si son sufridos más tempranamente y por los que se ha recibido algún tipo de presión para silenciarlos.

De acuerdo con los diferentes estudios (Davidson, Hughes y Blazer, 1991; Helzer, Robins, y McEvoy, 1987; Norris, 1992), se sabe que las mujeres corren un riesgo más alto que los hombres de desarrollar un TEPT, a pesar de exponerse con menor frecuencia a las experiencias traumáticas. También en la infancia se constatan estudios que muestran -en general- a las niñas más sintomáticas que los niños, como los de Giaconia et al en 1995 y los de Shannon et al, en 1994. Se ha indicado que la edad en la que ocurre un evento traumático, es un factor significativo para el desarrollo del TEPT, así Briere, Woo y McRae, Foltz y Sitzman (1997) apuntan que las experiencias traumáticas en la infancia se asocian con un mayor riesgo y con síntomas más severos.

En el DSM-IV-TR (American Psychiatric Association, 2000), se indica que el TEPT aparece cuando la persona ha sufrido "o ha sido testigo de" un acontecimiento estresante y extremadamente traumático, y donde el individuo se ve envuelto en hechos que representan un peligro real para su vida o cualquier otra amenaza para su integridad física. La respuesta del sujeto a este acontecimiento debe incluir temor, desesperanza y horror intenso (o en los niños/as, un comportamiento que se presenta como desestructurado o agitado).

El cuadro sintomático característico, secundario a la exposición al trauma, debe incluir la 
presencia de una continua reexperimentación del hecho traumático, incluso después de que éste se acabe, por ejemplo: flashbacks, pesadillas, pensamientos intrusivos y malestar emocional y fisiológico al recordar el evento; evitación de recuerdos del evento (evitación de personas, lugares y actividades que están asociados con el evento); interés disminuido en actividades que antiguamente resultaban placenteras; sentimientos de indiferencia, con un sentido de futuro limitado y exhibición de signos de arousal persistente (como dificultades para dormir, irritabilidad incrementada, problemas de concentración, examen continuo del ambiente por idea de peligro y respuesta de sobresalto aumentada).

\section{LOS ESTUDIOS SOBRE TEPT EN LA INFANCIA Y ADOLESCENCIA}

La ambigüedad conceptual y etiológica que rodea a este síndrome se acrecienta cuando nos centramos en la población infantil, según exponen Sarmiento et al (2006). Así, no es hasta la versión revisada del DSM-III (APA, 1987) cuando se considera que las reacciones de los niños al trauma pueden diferir de la de los adultos. Terr, en los Estados Unidos (1981, 1983) fue la primera en describir, de un modo más sistemático, las reacciones de los niños después de desastres.

En las delimitaciones del trastorno y según los hallazgos, se plantea la configuración de una tríada sintomática compuesta por:

(a) la re-experimentación del acontecimiento traumático en los menores tiende a reflejarse en una representación de recuerdos recurrentes e invasores del acontecimiento, los cuales se pueden expresar en pensamientos, percepciones o juegos repetitivos con elementos del trauma; también se presentan sueños recurrentes que en los menores pueden ser sobre el evento traumático o sueños terroríficos acerca de otras situaciones o también con un carácter más inespecífico (APA, 1995; March, Amaya y Pynoos, 1997; Márquez, 2000);

(b) la sensación de estar reviviendo el hecho traumático, puede expresarse en niños/as como re-escenificaciones del evento traumático, como ensoñaciones diurnas, alucinaciones e incluso estados disociativos; en niños/as muy rara vez se observan los flashbacks descritos en adultos (American Academy of Child and Adolescent Psychiatry, 1998; APA, 1995, Montt y Hermosilla,
2001 y Terr, 1985); y

(c) por último, los menores pueden presentar malestar psicológico severo y/o respuestas fisiológicas al exponerse a estímulos internos (recuerdos, sensaciones) o externos (lugares, fechas, eventos) que simbolicen o recuerden el evento traumático (American Academy of Child and Adolescent Psychiatry, 1998 y APA, 1995).

Luxenberg, Spinazzola, y van der Kolk (2001) señalan que, las investigaciones realizadas en las últimas dos décadas, han establecido firmemente la alta prevalencia de TEPT y su impacto potencialmente debilitante sobre la vida de los individuos. La investigación epidemiológica ha mostrado que, la mayoría de las personas sufren -por lo menos- un hecho traumático durante su vida y hasta un cuarto de estos individuos desarrollarán finalmente un TEPT(Davidson et al, 1991; Hidalgo y Davidson, 2000).

Los estudios basados en la población general de EEUU revelan tasas de prevalencia para el TEPT de -aproximadamente- un $8 \%$ de la población adulta. No existe actualmente información disponible referente a la prevalencia en la población general en España (Sarmiento et al, 2006).

Son pocos los estudios que han examinado los índices de exposición y el desarrollo del TEPT en niños y adolescentes, en población general. Los resultados de estos estudios indican que entre el $15-43 \%$ de chicas y el $14-43 \%$ de chicos han experimentado por los menos un evento traumático durante su vida -en los EEUU- (Nacional Center for PTSD, 2006). De los menores y adolescentes que han experimentado un trauma, el 3-15\% de las chicas y el $1-6 \%$ de los chicos cumplen criterios para TEPT. Trabajos como el de Norris, Foster y Weisshaar (2002), indican que las chicas tienen más riesgo de desarrollar TEPT que los chicos similarmente expuestos. En muestras escolares, algunos estudios han determinado tasas de exposición al trauma de entre el 40-70\% (Giaconia et al, 1995; Jenkins y Bell, 1994). Particularmente interesante resultan las altas tasas de exposición a traumas entre jóvenes hospitalizados con serios trastornos emocionales, siendo de hasta un 93\%, según refieren Lipschitz et al (1999). A raíz de estos porcentajes, algunos autores han coincidido en sugerir que la exposición a sucesos traumáticos puede conducir a serios trastornos de estrés postraumático, con tasas que oscilan entre el $10-100 \%$ de probabilidad 
(Sauter y Franklin, 1998; Stuber et al, 1991).

En un reciente estudio longitudinal de 1420 menores de 9, 11 y 13 años, Copeland et al (2007) encontraron que más de dos tercios de los menores informaron de un evento traumático después de los 16 años, desarrollando un $13,4 \%$ de estos niños/as algunos síntomas de estrés postraumático y menos del $0,5 \%$ cumplieron los criterios para TEPT según el DSM-IV. En definitiva, la variedad de tasas de prevalencia dependen de determinados factores de riesgo como la exposición a sucesos traumáticos, la severidad del suceso traumático, el procesamiento cognitivo del trauma (Brown, 2003), las características del menor/adolescente, la historia del trauma e historia psiquiátrica del menor, la influencia familiar (Buka et al, 2001; Fletcher, 2003; Fremont, 2004) o los criterios diagnósticos utilizados.

\section{EVALUACIÒN DEL TEPT}

Teniendo en cuenta las diferencias evidenciadas en cuanto a la semiología del Trastorno por Estrés Postraumático, es lógico pensar que la evaluación e intervención de estos pacientes no puede plantearse de igual modo que en el caso de la población adulta (Sarmiento et al, 2006). Existen serias dificultades metodológicas a la hora de evaluar determinados factores del TEPT en la infancia, tales como: dificultades para contar con la experiencia subjetiva; criterios diagnósticos para el TEPT que pueden no estar bien formulados para su aplicación en niños/as (Tierney, 2000); la evaluación del trauma carece de medidas psicométricas refinadas; las propias características de los síntomas del TEPT pueden afectar a la certeza y validez de las medidas estimadas del trauma.

Muchos de los estudios -la gran mayoríase han llevado a cabo con muestras clínicas, pero muy pocos han utilizado un grupo control; a la vez que, gran cantidad de esos estudios específicos, ha sido realizados con muestras pequeñas, lo que hace aún más difícil la generalización y aún cierta comparación de resultados (McCloskey, 2000); finalmente, pocos estudios incorporan en sus diseños el rigor empírico necesario para explorar la influencia de posibles variables moduladoras (por ejemplo, la edad o el género) o efectos mediadores como el apoyo de la familia (Cohen, Berliner y Mannarino, 2000).

Tal y como nos sugiere el estudio de Feeny et al (2004), una de las herramientas de evaluación de este trastorno más útil para los clínicos, junto con la entrevista estructurada -en población infantil- pueden ser los autoinformes, como los que se desarrollaran para el estudio de la psicopatología infantil y adolescente por los equipos de Achenbach et al (1991 a,c; 1989; 1995; 1997; 1998; 2001 a y b; 2002) y en España y Latinoamérica López Soler (López Soler et al, 1999 y López Soler, 1995; Daset, 1999 y 2002) donde además se incluye la estrategia de trabajo multi-informante (Achenbach, 1991b). El problema surge cuando comprobamos que, existen muy pocas medidas de autoinforme de síntomas infantiles de TEPT que hayan sido validadas psicométricamente e incluidas en los criterios del DSM-IV-TR (Saigh, 2004).

La mayoría de escalas relacionadas con el trauma para niños y adolescentes, son relativamente nuevas y fueron desarrolladas tanto para evaluar características relacionadas con síntomas de trauma, como, para medir el trauma en sí. Como muchas de estas escalas son tan recientes, no han sido lo suficientemente bien examinadas psicométricamente y las bases de datos no son todavía muy extensas, aunque van en aumento (Balaban, 2006; Ohan, Myers y Collett 2002). Se puede concluir que, en el campo de la evaluación de respuestas al trauma -en niños y adolescentes- hay instrumentos adecuados, pero es esencial más investigación. Algunas de las escalas que son utilizadas para medir TEPT en menores son: Impact of Event Scale (IES), (Horowitz, M et al, 1979; Creamer et al, 2003); The Children's Impact of Traumatic Events Scale (CITES-R), (Wolfe el al, 1992); The Child PTSD Symptom Scale (CPSS), (Foa, Edna B, 2001) y The revised version of the Screen for Child Anxiety Related Emotional Disorders (SCARED-R), (Muris, P. et al ; 1999).

\section{ESTUDIOS SOBRE TEPT EN DISTINTAS ETAPAS VITALES}

Numerosos estudios han mostrado que el TEPT consistentemente coocurre con otros trastornos. Estudios en población general y clínica informan que, aproximadamente de 50 a $90 \%$ de los individuos con TEPT crónico, tienen trastornos psicológicos comórbidos (Davidson et al, 1985; Green et al, 1992; Lerer et al, 1987; Shore, Vollmer y Tatum, 1989; Sierles et al, 1983).

El estudio nacional de comorbilidad en EEUU 
(Kessler et al, 1995) encuentra que aproximadamente el $84 \%$ de personas con TEPT tuvo otro diagnóstico de patología psíquica a lo largo de su vida, siendo el TEPT típicamente el trastorno primario. Además, mientras aproximadamente un quinto de todas las personas diagnosticadas con TEPT no cumplieron los criterios para otro trastorno, los restantes $79 \%$ cumplían criterios para al menos 1 trastorno adicional y un $44 \%$ cumplía los criterios al menos para otros 3 diagnósticos.

En relación al género, el $59 \%$ de los hombres y el $44 \%$ de las mujeres con TEPT reúnen criterios para tres o más diagnósticos psiquiátricos. Según Friedman (1996), la comorbilidad se suele dar con los siguientes trastornos: Afectivos (26-65\%), de Ansiedad (30-60\%), Alcoholismo o Abuso de drogas (60-80\%) o de Personalidad (40-60\%). En niños y adolescentes con TEPT, las comorbilidades comunes incluyen trastornos del apego, habilidades sociales deterioradas, agresividad, problemas en el control de los impulsos, ansiedad, depresión, somatización y uso de sustancias (Kessler, Borges, y Walters, 1999). De hecho, en una declaración consensuada, expertos en la materia asentaron que la "forma pura" de TEPT no es actualmente representativa de la presentación clínica típica de TEPT (Ballenger et al, 2000). En la literatura sobre TEPT, los problemas psicológicos que no caen dentro de la estructura de TEPT son generalmente referidos como "condiciones comórbidas", como si ocurrieran independientemente de los síntomas del TEPT, como corroboran van der Kolk et al, en 2005.

Al parecer, la cultura popular está cada vez más concienciada sobre el trauma, sin embargo un gran número de investigadores que estudian los efectos del trauma sobre el funcionamiento psicológico (Breslau, Davis y Andreski, 1991; Cole y Putnam, 1992; Luxenber, Spinazzola y van der Kolk, 2001) han mostrado que el TEPT recoge sólo un limitado aspecto de la psicopatología postraumática y especialmente en menores (por ejemplo Brett, Spitzer y Williams, 1988; Briere, 1987; Cole y Putnam, 1992; Scheeringa et al, 1995; Scheeringa et al, 2003; Summit, 1983; Terr, 1979).

Como señalan Cook et al (2005) y Van der Kolk (2005), el diagnóstico de TEPT no captura los efectos del desarrollo en la exposición a trauma complejo. Ya que, si en cualquier época del desarrollo de la persona, la presencia de acontecimientos estresantes o situaciones vitales adversas, es importante en relación a la salud física y psicológica, durante la infancia su impacto puede ser dramáticamente significativo; ya que no va a afectar a un ser humano biológica, psicológica y socialmente maduro, sino a un ser humano en una fase de desarrollo que requiere ciertas condiciones externas, de estabilidad y protección (Lòpez Soler, 2008, en prensa).

Clínicos e investigadores, entre ellos Briere (1987, 1992), Courtois (1988), Finklehor (1984) y Herman (1992 a, 1992 b) argumentaron que el diagnóstico TEPT no fue adecuado para las reacciones experimentadas por víctimas de abuso infantil y trauma doméstico y otras poblaciones, donde la traumatización ocurrió de forma repetida y/o extensa. Individuos expuestos a trauma en varios momentos de su vida y en periodos de desarrollo, padecieron una variedad de problemas psicológicos no incluidos en el diagnóstico de TEPT, incluyendo depresión, ansiedad, odio a si mismo, disociación, abuso de sustancias, comportamientos autodestructivos y de riesgo, revictimización, dificultades en las relaciones interpersonales e Intimas, problemas médicos y somáticos y desesperanza (Courtois, 2004).

\section{MENORES QUE HAN SUFRIDO ABUSO Y NEGLIGENCIA; UNA PARTICULAR CON- FIGURACIÒN DEL TEPT: EL TEPT COMPLEJO O DESNOS}

Los resultados del grupo de trabajo del DSMIV para TEPT, sugieren que, el trauma tiene su más profundo impacto cuando su comienzo ocurre durante la temprana infancia o adolescencia, y provoca un daño más penetrante que con un comienzo tardío (van der Kolk et al, 1996; van der Kolk, 1985). Desde estos estudios iniciales, investigaciones sobre una variedad de poblaciones y escenarios, han encontrado apoyo para la hipótesis de que, el trauma interpersonal temprano, especialmente el de abuso en la infancia, presenta un riesgo más alto para el desarrollo de un TEPT complejo o DESNOS ${ }^{1}$ (Trastorno por Stress extremo no expecificado, por sus siglas en inglés) que los accidentes de tráfico y desastres (Roth et al, 1997).

El abuso y negligencia en la infancia son extremadamente comunes en nuestras so- 
ciedades y sus efectos persisten a través del tiempo. Cada año, alrededor de 3 millones de menores son atendidos por abuso-negligencia en los EEUU (Wang y Daro, 1997). En España hay más de 30.000 niños y niñas tutelados por organismos públicos, de los que 14.600 menores viven en centros de acogida; en torno a 15.900 lo hacen con parientes o en acogimiento familiar administrativo; y 2.800 en régimen de acogimiento familiar judicial. Estos menores suelen recibir una variedad de etiquetas psicológicas, pero ninguno de estos diagnósticos recoge sus profundas alteraciones en el desarrollo, ni los orígenes traumáticos de sus particulares presentaciones clínicas (van der Kolk, 2001). Van der Kolk en el año 2005, pone énfasis en el papel crucial que juega la familia, en determinar cómo son las experiencias infantiles y cómo se recuperan los menores de una experiencia traumática. Cuando la relación con la familia es la fuente del trauma, pueden verse severamente alteradas muchas competencias críticas del desarrollo. El abuso o maltrato íntimo, doméstico o familiar, se da durante largos periodos de tiempo, en los cuales los menores quedan atrapados -y entrampados-, a la vez que condicionados por un gran número y variedad de circunstancias estresantes. Debido a que la víctima es psicológica y físicamente inmadura, su desarrollo queda seriamente comprometido por el abuso repetido y la respuesta inadecuada, ya sea por parte de algunos miembros de la familia o de otros cuidadores, por ejemplo cuando la madre niega o acepta la relación incestuosa del padre con una hija (Lòpez Soler, 2008; en prensa). Modelos de apego inseguro, han sido consistentemente documentados en hasta un $90 \%$ de maltrato a niños. Otros factores tales como, adversidades socioeconómicas, conflictos familiares, psicopatología o adicción del cuidador, contribuyen más a reacciones de trauma complejo en el niño. Si los niños son expuestos a un estrés difícil de controlar y si el cuidador no asume la función de modular el arousal del niño, como ocurre cuando los niños se exponen a desestructuración o violencia familiar, ellos -por sí mismos- son incapaces de organizar la experiencia de un modo coherente, ya que ellos dependen de sus cuidadores para su propia supervivencia y no tienen la opción de informar o apartarse del conflicto (van der Kolk, 2005). No resulta difícil comprender, que estos menores incorporen el sistema de creencias del agresor de modo defensivo (Síndrome de
Estocolmo), ya que no han tenido otra posibilidad de estructurar su mapa de conocimiento y experiencias cognitivas y afectivas (López Soler, 2008; en prensa).

\section{PERFIL SINTOMÀTICO DEL DESNOS}

El perfil de la sintomatología DESNOS, ha sido identificado en muchos estudios e investigaciones clínicas y ha recibido atención generalizada en el campo del trauma en los recientes años. El grupo de trabajo del TEPT del DSM-IV, identificó alteraciones en 6 dominios de funcionamiento (Tabla 1), siendo necesarios para el diagnóstico de DESNOS, que se cumpla con los criterios explicitados a continuación:

- $\quad$ Alteración en la regulación de afectos e impulsos: incluyendo dificultad con la modulación de la ira y autodestructividad. De hecho, se ha sugerido que la disregulación del afecto puede ser la disfunción central del trauma psicológico. Se atemorizan fácilmente, parecen tener reacciones "extremas" a estímulos neutrales o moderados, tienen problemas para calmarse y pueden usar medidas extremas autodestructivas, tales como autolesión, abuso de drogas, trastornos alimentarios o actividad sexual compulsiva, en un intento de manejar sus emociones (Felitti et al, 1998; Linehan et al, 1994).

- $\quad$ Alteraciones en atención y consciencia: llevando a amnesias y episodios disociativos $y$ des-personalización.

- Alteraciones en la auto-percepción: tales como una crónica sensación de culpa y responsabilidad y sentimientos de vergüenza crónicos. Con frecuencia desarrollan visiones negativas sobre ellos mismos, se sienten impotentes, inútiles e indeseables a los demás. Estas percepciones surgen directamente de la forma en la que el niño pequeño interpreta el mundo, llevándoles a creer que ellos han "causado" su propio maltrato (Herman, 1992).

- Alteraciones en la relación con los otros: dificultades para confiar en otros, incapacidad para sentir intimidad con los demás, re-victimizaciones y la victimización de otros (Fleming et al, 1999; Bryer et al, 1987; Lisak, Koper y Song, 1996).

- Somatización y/o problemas médicos: sufren dolencias físicas sin explicación médica. Hay una amplia evidencia de que las experiencias traumáticas repetidas tienen un impacto sobre el nivel biológico. Investigaciones como 
las de Pitman et al (1987), Shalev y RogelFuchs (1993), van der Kolk (1996) y Yehuda (1999) han demostrado que la respuesta de estrés implica la liberación de endógenos, hormonas sensibles al estrés. Tienen dificultades para ajustar sus niveles de arousal fisiológico, sugiriendo que el sistema nervioso tiene una mayor respuesta a estímulos previamente inocuos.

- Alteración en el sistema de significados: con frecuencia sienten desesperanza sobre encontrar a alguien que entienda su sufrimiento. Suelen informar de que su vida no tiene sentido (Herman, 1992). Una profunda sensación de impotencia aprendida, adoptan una actitud fatalista de la vida, anticipando que no serán capaces de hacer cambios positivos en su vida.

Se puede considerar que, algunas de estas características suponen mecanismos adaptativos que estructuran el significado del mundo y

\begin{tabular}{|c|c|}
\hline \multicolumn{2}{|c|}{$\begin{array}{l}\text { Tabla 1: } \\
\text { SINTOMAS DEL TRASTORNO POR ESTRÉ } \\
\text { POSTRAUMÁTICO COMPLEJO_(DESNOS) }\end{array}$} \\
\hline \multirow{6}{*}{$\begin{array}{l}\text { AFEC:LOS E } \\
\text { IMPUI.SOS }\end{array}$} & Regulacion del Afecto \\
\hline & Modulación de la lra \\
\hline & A utod estruetividad \\
\hline & Preocupacion Suicida \\
\hline & $\begin{array}{l}\text { Dificultad elr } \\
\text { modulación de la } \\
\text { sexualidad }\end{array}$ \\
\hline & Conductas de riesgo \\
\hline \multirow{3}{*}{$\begin{array}{l}\text { ATTNOCION Y } \\
\text { CONCIENCIA }\end{array}$} & Annosit] \\
\hline & $\begin{array}{c}\text { Fpisodios disociativos } \\
\text { transilonios }\end{array}$ \\
\hline & Despersonalización \\
\hline \multirow{5}{*}{$\begin{array}{l}\text { AUTOLLLR- } \\
\text { CTFCTCDN }\end{array}$} & Incficacia \\
\hline & $\begin{array}{c}\text { Colpa y } \\
\text { Responsabilidad }\end{array}$ \\
\hline & Verğtienza \\
\hline & Incomprension \\
\hline & $\begin{array}{l}\text { Minimización } \\
\text { personal }\end{array}$ \\
\hline \multirow{3}{*}{$\begin{array}{l}\text { RELACIONES } \\
\text { INTTRPTRSO- } \\
\text { NALES }\end{array}$} & $\begin{array}{l}\text { Inlabilidud para las } \\
\text { relaciones fntimas }\end{array}$ \\
\hline & Revictimización \\
\hline & Victimizar a otros \\
\hline \multirow{5}{*}{$\begin{array}{l}\text { SOMAII/A- } \\
\text { CIONTS }\end{array}$} & Sistema digestivo \\
\hline & Dolor crónico \\
\hline & $\begin{array}{l}\text { Sintomas } \\
\text { cardiopulmona res }\end{array}$ \\
\hline & $\begin{array}{l}\text { Símlomàis de } \\
\text { conversión }\end{array}$ \\
\hline & Sistema reproductor \\
\hline \multirow{3}{*}{$\begin{array}{l}\text { SLIEMA DE } \\
\text { STCINTTCAT) }\end{array}$} & In felicidad \\
\hline & $\begin{array}{l}\text { Perdida del Sistema } \\
\text { previo de Creencias }\end{array}$ \\
\hline & Devesperanka \\
\hline
\end{tabular}

de las relaciones, en un proceso de aprendizaje patológico, que está siendo legitimado por el resto de las personas consentidoras (López Soler, 2008; en prensa).

Las consecuencias postraumáticas varían ampliamente, por lo tanto, el enfoque inicial para la evaluación es críticamente importante (Briere y Spinazzola, 2005). Es difícil realizar una evaluación de las posibles variables psicológicas afectadas en menores que han sufrido maltrato grave y continuo, y por tanto, un posible trauma complejo (López Soler et al, 2007 a y b).

El clínico debe considerar el amplio rango de respuestas potencialmente traumáticas, atribuibles a la historia de un determinado paciente y sus factores de riesgo. En muchos casos, esto puede requerir de la administración de tests psicológicos de amplio espectro, tanto genéricos como otros más específicos de trauma, además de otros tests relevantes según la presentación clínica específica del individuo.

Para Briere y Spinazzola (2005), la mayoría de tests de consecuencias postraumáticas complejas, miden la sintomatología como variables continuas (opuestas a diagnósticos dicotómicos), así que la evaluación puede ayudar a determinar la extensión de los síntomas postraumáticos, más que la presencia o ausencia de un trastorno DSM-IV solamente.

Una evaluación exhaustiva de trauma complejo incluye información de un número importante de fuentes, incluyendo las propias revelaciones del niño o adolescente, informes colaterales de cuidadores y otras personas significativas, las observaciones del terapeuta y medidas de evaluación estandarizadas. Las técnicas de evaluación estandarizadas, que son culturalmente sensibles y apropiadas al lenguaje, son completadas por el paciente, cuidador y -si es posible- por el docente del niño (Briere y Spinazzola, 2005).

La evaluación debe señalar tanto la exposición a trauma complejo, como los resultados postraumáticos complejos. Éstos deben ser investigados, además, a partir de la historia de desarrollo, historia familiar, historia del trauma para el niño y la familia, relaciones de apego primarias, servicios protectores implicados, emplazamiento de la historia, enfermedades mentales parentales-familiares, abuso de sustancias, historia legal, habilidades de afrontamiento, fortalezas del niño o adolescente y familia, estresores ambientales (Cook et al, 2005). Si es complicada la evaluación del TEPT 
en la infancia, igualmente difícil resulta encontrar instrumentos de evaluación adecuados y psicométricamente fiables para analizar los diferentes síntomas del Trauma Complejo (López Soler, 2008; en prensa). Hasta la fecha, la evaluación usando instrumentos desarrollados para criterios TEPT puede, desafortunadamente, no cubrir la complejidad de pacientes con DESNOS (Courtois, 2004). A pesar de esto, según refieren Briere y Spinazzola (2005), la última década ha sido testigo del desarrollo y crecimiento de un gran número de tests -validados psicométricamente- y entrevistas que evalúan consecuencias relacionadas con el trauma. Dos instrumentos clínicos han sido desarrollados y validados para el propósito de evaluación exhaustiva del DESNOS, en la práctica clínica y en investigación. Estos son: la Entrevista Estructurada del Trastorno de Estrés Extremo (SIDES) y el Inventario Autoinformado del Trastorno de Estrés Extremo (SIDES-SR).

EI SIDES (Pelcovitz et al, 1997) fue desarrollado por el grupo de trabajo del DSM-IV y fue diseñado para medir presencia de DESNOS -actual y anterior- y para describir la severidad de la sintomatología actual (último mes). Investigaciones sobre sus propiedades psicométricas han apoyado su fiabilidad como un instrumento diagnóstico (Pelcovitz et al, 1997) y como una medida continua de severidad de síntomas para el diagnóstico en conjunto (Spinazzola, Blaustein, van der Kolk, datos no publicados, 2001). Investigaciones adicionales que han usado este instrumento, han proporcionado -preliminarmente- sólidas evidencias empíricas para la validez del constructo de DESNOS (Zlotnick y Pearlstein, 1997; Blaustein et al, 2000).

EI SIDES-SR (Spinazzola et al, 2001) es una técnica de autoinforme que ha sido desarrollada más recientemente, para la medida de la línea de base de severidad del DESNOS; línea de base de la severidad de cada uno de los grupos de síntomas individuales y cambio de síntomas a través del tiempo.

Las historias de agresiones físicas y sexuales en la infancia, también están asociadas con una multitud de otros problemas psicológicos en la adolescencia y edad adulta: abuso de sustancias, personalidad límite y antisocial, así como, trastornos alimentarios, disociativos, afectivos, somatoformes, cardiovasculares, metabólicos, inmunológicos y sexuales (Breslau et al, 1997; Cloitre et al, 2001; Dube et al, 2001; Felitti et al, 1998; Finkelhor y Kendall-Tackett, 1997; Her- man, Perry y van der Kolk, 1989; Kilpatrick et al, 2000, Kilpatrick et al, 2003; Margolin y Gordis, 2000; Putnam y Trickett, 1997; van der Kolk, Perry y Herman, 1991; Wilson et al, 1999; Zlotnitk et al, 1996). Está claro que, estas historias no sólo pueden producir secuelas duraderas en sí mismas, sino que también son un factor de riesgo para ser revictimizado en el futuro (Neumann et al, 1996) y para responder más tarde a eventos traumáticos con síntomas más severos y complejos (Bremner et al, 1993). Otra consecuencia a largo plazo del maltrato es la violencia transgeneracional; un niño maltratado tiene alto riesgo de ser perpetrador de maltrato en la etapa adulta, a su pareja o a sus hijos, mientras que en las niñas está menos clara su evolución (López Soler, 2008; en prensa).

En contraste con el grupo de trabajo del DSM-IV, el cual encontró un $92 \%$ de comorbilidad entre DESNOS y TEPT, Ford y Kidd, (1998) han confirmado que la presencia de TEPT no es una condición necesaria previa para DESNOS. Esta cuestión requerirá más investigación empírica (Luxenberg, Spinazzola y van der Kolk, 2001).

\section{DESARROLLO DE INSTRUMENTOS DE EVALUA- CIÓN Y RESULTADOS PRELIMINARES DEL EQUI- PO ESPAÑOL}

El campo de los estudios de estrés traumático está todavía en su infancia y predomina más el desconocimiento que el conocimiento. Por este motivo, es necesario usar con cuidado las formulaciones teóricas y avanzar con precaución. Son necesarios muchos más estudios para dar luz a estos asuntos complejos (Dietrich, 2000). Ford, uno de los expertos en el tratamiento del trauma infantil, describió los objetivos para el futuro que incluyen: recopilar estudios de caso de menores que hayan experimentado trauma profundo, proporcionados por clínicos nacionales e internacionales; refinar los criterios para el diagnóstico de trauma complejo y desarrollar instrumentos basados en entrevistas y escalas que los clínicos puedan usar en su práctica diaria (Moran, 2007).

Nuestro equipo, está realizando las evaluaciones y tratamientos psicológicos de menores maltratados y tutelados por la Comunidad Autónoma de Murcia y la sintomatología encontrada es más compatible con la existencia de reacciones postraumáticas complejas, que afectan a todo el desarrollo personal que, con 
una sintomatología específica de TEPT (Tabla 2).

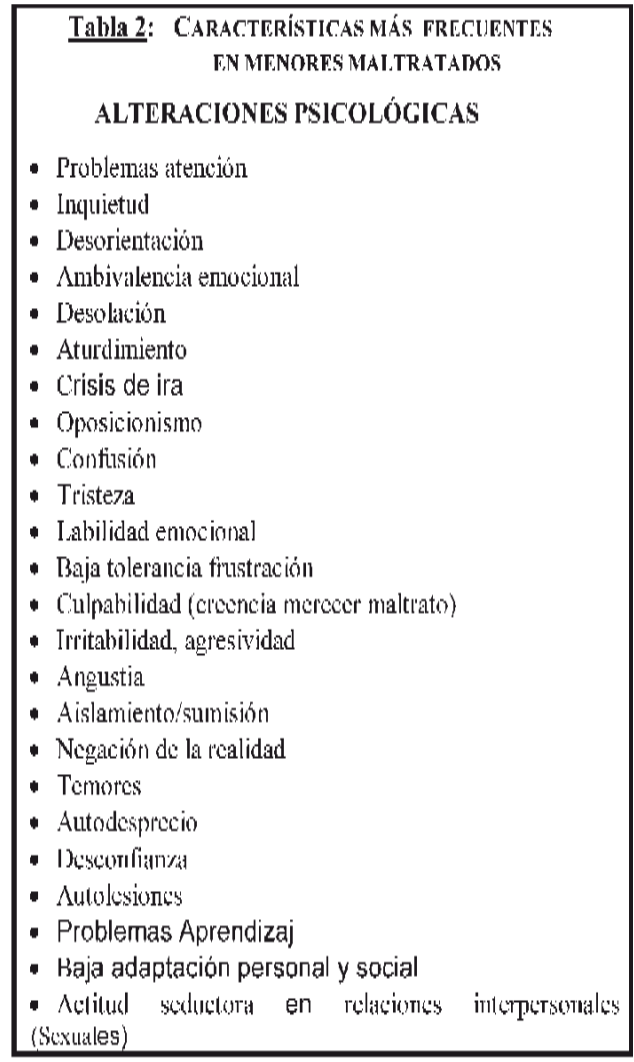

Lòpez Soler, 2008 (en prensa)

En este sentido, se elaboró un Listado para el clínico de Indicadores de Trauma Complejo (publicado inicialmente en la Revista de Psicopatología y Psicología Clínica, López Soler, 2008); el mismo permitió reunir información significativa sobre las características de los menores que han sufrido trauma grave y crónico durante su infancia; algunos de los datos muestran que: mas del $40 \%$ sufren alteraciones en la regulación de los afectos (94\%) y en el control de los impulsos (68\%), de la consciencia (70.6\%), en la autopercepción (61.8), en la percepción del/la maltratador/a (52\%), en las relaciones con pares (65\%) y con acogedores/ cuidadores $(73,5 \%)$, en las relaciones intimas (41\%), informan sobre problemas depresivos (50\%), de ansiedad (67.5\%), y desesperanza (59\%). La puntuación del Coeficiente Alfa de este listado es de .835 .

Considerando la importancia de realizar una evaluación fiable y válida, se ha desarrollado un protocolo de evaluación (Tabla 3), que permita discernir adecuadamente la sintomatología característica de los menores con traumas graves; para esto se ha administrado antes y después del tratamiento un conjunto de técnicas que exploran la sintomatología postraumática y los efectos del tratamiento. De esta manera, se busca reconocer tanto la sintomatología propia de TEPT, como la configuración del DESNOS o TEPTC (Trastorno por Estrés Postraumático Complejo) en la infancia.

\section{CONCLUSIONES}

El abordaje de un trastorno por estrés postraumático en la infancia y adolescencia supone, sin lugar a dudas, un camino largo y esforzado. El solo hecho de enfrentar temáticas como el abuso, maltrato y negligencia en edades tempranas, hace indispensable una incorporación de la mirada de las Psicologías del Desarrollo, de la Personalidad y de la Psicopatología y además, de toda el área de la Evaluación Psicológica.

Una especial consideración merece el fenómeno del cambio, como denominador común, tanto de lo general del menor como de lo particular del trastorno, que también acompasa -muchas veces- ese crecimiento. Todo esto pone de relieve nuevamente que, el estudio de la patología en la infancia y adolescencia no puede pensarse en términos de gnosografias adultas, sino que demanda una especificidad tanto evaluativa como de intervención.

EI DESNOS, en su carácter de complejo, a primera vista puede impresionar como difuso, pero poco a poco va develando sus síntomas característicos, para dejar de ser una categoría accesoria para convertirse una entidad definida. El Protocolo de Evaluación que se presenta busca atender esas complejidades de un trastorno que supone un alto nivel de sufrimiento y donde son tantas las variables a analizar y los factores a considerar.

Una investigación rigurosa, con instrumentos validados psicométricamente y un acervo de casos en diferentes culturas y sistemas de creencias, además de la imprescindible comunicación entre los expertos, será sin duda el camino que posibilite una mayor y mejor definición de las estrategias de diagnóstico e intervención.

\section{REFERENCIAS}

Achenbach, T. M. (1991a). Manual for the Child 


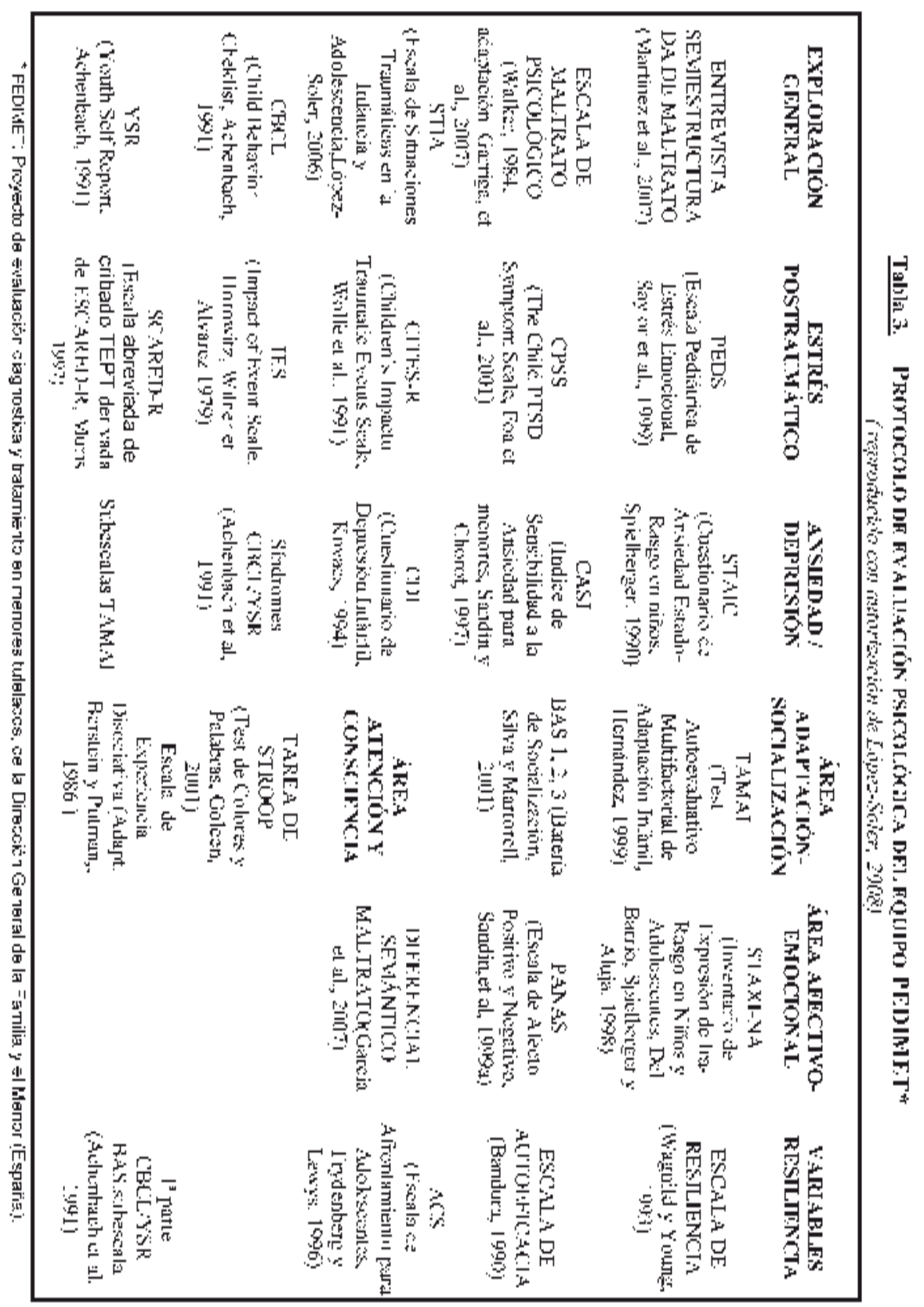


Behavior Checklist/4-18 and 1991 Profiles. Burlington: University of Vermont, Department of Psychiatry.

Achenbach, T. M. (1991b). Manual for the Teacher's Report Form and 1991 Profiles. Burlington: University of Vermont, Department of Psychiatry.

Achenbach, T. M. (1991c). Manual for the Youth SelfReport and 1991 Profiles. Burlington: University of Vermont, Department of Psychiatry.

Achenbach, T. M., \& McConaughy, S. H. (1997). Empirically based assessment of child and adolescent psychopathology (2nd ed., Vol. 13). Thousand Oaks, CA: Sage.

Achenbach, T. M., \& Rescorla, L. A. (2001a). Manual for ASEBA School-Age Forms \& Profiles. Burlington, VT: University of Vermont, Research Center for Children, Youth, \& Families.

Achenbach, T. M., Conners, C. K., Quay, H. C., Verhulst, F. C. \& Howell, C. T. (1989). Replication of empirically derived syndromes as a basis for taxonomy of child adolescent psychopathology. Journal of Abnormal Child Psychology, 17, 299/323.

Achenbach, T. M., Howell, C. T., McConaughy, S. H., \& Stanger, C. (1998). Sixyear predictors of problems in a national sample: IV. Young adult signs of disturbance. J. of the American Academy of Child and Adolescent Psychiatry, 37, 718-727.

Achenbach, T., Dumenci, L. \& Rescorla, L.A. (2002). Ten-year comparisons of problems and competencies for national samples of youth: self, parent, and teacher reports. Journal of Emotional and Behavioral Disorders, 4, 215-228.

Achenbach, T. M. \& Dumenci, L. (2001b). Advances in Empirically Based Assessment: Revised CrossInformant Syndromes and New DSM -Oriented Scales for the CBCL, YSR, and TRF: Comment on Lengua, Sadowksi, Friedrich, and Fisher. Journal of Consulting and Clinical Psychology:69 (4), 699-702

Achenbach, T. M. (1995). Empirically Based Assessment and Taxonomy: Applications to Clinical Research. Psychological Assessment: 7 (3), 261-274.

American Academy of Child and Adolescent Psychiatry (1998). Practice parameters for the assessment and treatment of children and adolescents with posttraumatic stress disorder. Journal of American Academy of Child and Adolescent Psychiatry, 37 suppl: 4-26.

American Psychiatric Association (APA). (1987). Diagnostic and statistical manual of mental disorders (DSM-III-R.) Washington, DC: APA.

American Psychiatric Association (APA). (2000). Diagnostic and statistical manual of mental disorders: DSM-IV-TR. ( $1^{\circ}$ Ed.). Washington, DC: APA.

Asociación Americana de Psiquiatría. Manual diagnóstico y estadístico de los trastornos mentales, cuarta edición (DSM-IV). Barcelona, Masson
S.A., 1995.

Atkinson, R.M., Sparr, L.F.y Sheff, A.G. (1984). Diagnosis of posttraumatic stress disorder in Vietnam veterans: preliminary findings. American Journal of Psychiatry, 141, 694-969.

Balaban V. (2006). Psychological assess-ments of children in disasters and e-mergencies. Disasters, 30, 178-198.

Ballenger, J., Davidson, J., Lecrubier, Y., Nutt, D. J., Marshall, R.D., Nemeroff, C.B., Shalev, A.Y. y Yehuda, R. (2000). Consensus statement on posttraumatic stress disorder from the inter-national consensus group on depression and anxiety. Journal of Clinical Psychology, 61, 60-66.

Bandura, A. (1990). Multidimensional Scales of Perceived Self-efficacy. Stanford University, Stanford, C.A.

Bernstein, E.M. y Putnam, F.W. (1986). Development, reliability, and validity of a dissociation scale. Journal of Nervous and Mental Disease, 174, 727-735

Blaustein, M.E., Spinazzola, J., Simpson, W. y van der Kolk, B.A. (2000). Psychological sequelae of early trauma: Comorbid diagnoses or diagnostic entity? Paper presented at: 16th Annual Meeting of the International Society for Traumatic Stress Studies; November, San Antonio, TX.

Bremner, J.D., Southwick, S.M., Jonson, D.R., Yehuda, R. y Charney, D.S. (1993). Childhood physical abuse and combat-related PTSD in Vietnam veterans. American Journal of Psychiatry, 150, 235-239.

Breslau, N., Davis, G.C. y Andreski, P. (1991). Traumatic events and post-traumatic stress disorder in an urban population of young adults. Archives of General Psychiatry, 48, 216-222.

Breslau, N., Davis, G.C., Andreski, P., Peterson, E.L. y Schultz, L.R. (1997). Sex differences in posttraumatic stress disorder. Archives of General Psychaitry, 54 (11), 1044-1048.

Briere, J. (1987). Post-sexual abuse trauma: Data and implications for clinical practice. Journal of Interpersonal Violence, 2, 367-379.

Briere, J. (1992). Child abuse trauma: theory and treatment of the lasting effects. Newbury Park, CA: Sage.

Briere, J. y Spinazzola, J. (2005). Phenomenology and Psycho-logical Assessment of Complex Posttraumatic States. Journal of Traumatic Stress, 18 (5), 401-412.

Briere, J., Woo, R. y McRae, B. Foltz, J. y Sitzman, R. (1997). Lifetime victimization history, demographics, clinical status in female psychiatric emergency room patients. Journal of Nervous and Mental Disease, 185, 95-101.

Brown, E.J. (2003). Child physical abuse: Risk for psychopathology and efficacy of interventions. Current Psychiatry Reports, 5 (2), 87-94.

Bryer, J., Nelson, B., Millar, J.B. y Krol, P. (1987). Childhood sexual and physical factors in adult 
psy-chiatric illness. American Journal of Psychiatry, 144, 1426-1430.

Buka, S.L., Stichick, T.L., Birdthistle, I. y Earls, F.J. (2001). Youth exposure to violence: preva-lence, risks, and consequences. American Journal of Ortho-psychiatry, 71 (3), 298-310.

Cazabat, E. (2001). Trastorno por estrés postraumático. Criterios diagnósticos.Psicología online, 2.

Chiape V. (2000). Clínica de los trastornos por ansiedad: estrés postraumático y trastorno de pá-nico. En: Grau A. y Meneghello J. (eds). Psiquiatría y Psicología de la Infancia y Adolescencia. Buenos Aires, Editorial Médica Panamericana, 334-346.

Cloitre, M., Tardiff, K., Marzuk, P.M., Leon, A.C. y Portera, L. (2001). Consequences of childhood abuse among male psychiatric inpatients: Dual roles as victims and perpetrators. Journal of Traumatic Stress, 14 (1), 47-61.

Cohen, J.A., Berliner, L. y Mannarino, A.P. (2000). Treating trauma-tizing children: A research review and synthesis. Trauma, Violence y Abuse, 1, 29-47.

Cole, P. y Putnam, F.W. (1992). Effect of incest on self and social functioning: a developmental psychopathology perspective. Journal of Consulting and Clinical Psychology, 60, 174-184.

Cook, A., Spinazzola, J., Ford, J., Landtree, Ch., Blaustein, M., Cloitre, M., DeRosa, R., Hubbard, R., Kagan, R., Liataud, J., Mallah, K., Olafson, E. y van der Kolk, B. (2005). Complex Trauma in Children and Adole-scent. Psychiatric Annals, 35 (5), 390-398.

Courtois, C. (2004). Complex Trauma, Complex Reactions: Assessment and Treatment. Psychotherapy: Theory, Research, Practice, Training, 41 (4), 412-425

Courtois, C.A. (1988). Healing the incest wound: Adult survivors in therapy. New York: Norton.

Creamer M. Bell R. Failla S. (2003). Psychometric properties of the Impact of Event Scale - Revised. Behaviour Research \& Therapy. 41(12):148996,

Daset, L. R. ( 1999). Aproximación a una taxonomía empírica: estudio en jóvenes de Uruguay. Comunicación. /l Congreso de la Aso-ciación española de Psicología Clínica y Psicopatología. Libro de Resúmenes: 83-84. Murcia.

Daset, L.R. (2002). Depresión en la Adolescencia: una aproximación desde la evaluación empírica. Prisma, 18: 25-32.

Davidson, J.R.T., Swartz, M., Stork, M., Krishnan R.R., Hammett E.(1985). A diagnostic and family study of posttraumatic stress disorder. American Journal of Psychiatry, 142, 90-93.

Davidson, J.R.T., Hughes, D. y Blazer, D. y George, L.K. (1991). Posttraumatic stress disorder in the community; an epidemio-logical study. Psychologie Médi-cale, 21, 1-19.

Del Barrio, V., Spielberger, C.D. y Aluja, A. (2005). Inventario de expresión de ira estado-rasgo en niños y adolescentes, STAXI-NA. TEA Ediciones, S.A., Madrid.

Dietrich, A. (2000). As the Pendulum Swings: The Etiology of PTSD, Complex PTSD, and Revictimization. Traumatology, 6 (1), 41-59.

Dube, S.R., Anda, R.F., Felitti, V.J. y Chapman, D.P., Williamson, D.F. y Giles, W.H. (2001). Childhood abuse, household dysfunction, and the risk of attempted suicide throughout the life span: Findings from the Adverse Childhood Experiences Study. Journal of the American Medical Association, 286 (24), 3089-3096.

Feeny, N.C., Treadwell, K.R.H., Foa, E.B. y March, J. (2004). Posttraumatic stress disorder in youth: a critical review of the cognitive and behavioral treatment outcome literature. Professional Psychology: Research and Practice, 35 (5), 466476.

Felitti, V., Anda, R., Nordernbeg, D., Willianson, D.F., Spitz, A.M., Edwards, V., Koss, M.P. y Marks, J.S. (1998). Relationship of childhood abuse to many of the leading causes of death in adults: The adverse childhood experiences (ACE) study. American Journal of Preventive Medicine, 14, 143-166.

Finkelhor, D. y Kendall-Tackett, K. (1997). A developmental perspective on the childhood impact of crime, abuse and violent victimization. In D. Cicchetti \& S. Toth (Eds.), Rochester symposium on developmental psychopathology and developmental perspectives on trauma (pp. 1-32). Rochester, NY: University of Rochester Press.

Finklehor, D. (1984). Child sexual abuse: New theory and research. New York: Free Press.

Fleming, J., Mullen, P.E., Sibthrope, B. y Bammer, G. (1999). The long-term impact of childhood sexual abuse in Australian women. Child Abuse \& Neglect, 23, 145-159.

Fletcher, K.E. (2003). Childhood posttraumatic stress disorder. En E.J. Mash y R.A. Barkley. Child Psychopathology. New Cork: The Guildford Press.

Foa, E. B., Johnson, K. M., Feeny, N. C., Treadwell, K. R. H. (2001). The Child PTSD Symptom Scale: A preliminary examination of its psychometric properties. Journal of Clinical Child Psychology, 30, 376-384.

Foa, E.B. (2002). The Child PTSD Symptom Scale (CPSS). Available from Edna Foa, PhD, Center for treatment Study of Anxiety, University of Pennsylvania School of Medicine, Department of Psychiatry, 3535 Market Street, Sixth Floor, Phladelpha, PA 19104.

Ford, J.D. et al (2006). Disorders of Extreme Stress (DESNOS) Symptoms Are Associated With Type and Severity of Interpersonal Trauma Exposure in a Sample of Healthy Young Women . Journal of Interpersonal Violence, Vol. 21, No. 11, 13991416.

Ford, J.D. y Kidd, T.P. (1998). Early childhood trauma and disorders of extreme stress as predictors of 
treatment outcome with chronic posttraumatic stress disorder. Journal of Traumatic Stress, 11 (4), 743-761.

Fremont, W.P. (2004). Childhood reactions to terrorism-induced trauma: a review of the past 10 years. Journal of American Academy of Child and Adolescent Psychiatry, 43 (4), 381-392.

Friedman, M. (1996). PTSD Diagnosis and treatment for mental health clinicians. Community Mental Health Journal 32 (2), 173-189.

Frydenberg, E. y Lewis, R. (1996). Escalas de afrontamiento para adolescentes. ACS. TEA Ediciones, S.A., Madrid.

García Montalvo, C., López-Soler, C., Martínez, A. y Garriga, A. (2007). Diferencial semántico maltrato infantil. Documento no publicado.

Garriga, A., López-Soler, C., Martínez, A., Fernández, V., Castro, M. y Puerto, J.C. (2007). Escala de maltrato psicológico, adaptación de Escala de maltrato a mujeres, en L. Walter (1984). Universidad de Murcia. Documento no publicado.

Giaconia, R.M., Reinherz, H.Z., Silverman, A.B., Pakiz, B., Frost, A.K. y Cohen, E. (1995). Traumas and posttraumatic stress disorder in a community population of older adolescents. Journal of the American Academy of Child and Adolescent Psychiatry, 34, 1369-1380.

Golden, C.J. (2007). Test de colores y palabras, STROOP. TEA Ediciones, S.A., Madrid. (5 edición).

Green, B.L., Lindy, J.D., Grace, M.C. y Leonard, A.C. (1992). Chronic posttraumatic stress disorder and diagnostic comorbidity in a disaster sample. Journal of Nervous and Mental Disease, 180, 760-766.

Gurwitch, R., Sullivan, M. y Long, P. (1998). The impact of trauma and disaster on young children. Child and Adolescent Psychiatric Clinics of North America, 7, 19-32.

Helzer, J.E., Robins, L.N. y McEvoy, L. (1987). Posttraumatic stress disorder in the general population. New England Journal of Medicine, 317, 1630-1634.

Herman, J.L, Perry, J.C. y van der Kolk, B.A. (1989). Childhood trauma in borderline personality disorder. American Journal of Psychiatry, 22, 231-237.

Herman, J.L. (1992a).Complex PTSD: A syndrome in survivors of prolonged and repeated trauma. Journal of Traumatic Stress, 5, 377-391.

Herman, J.L. (1992b). Trauma and recovery: The aftermath of violence-From domestic to political terror. New York: Basic Books.

Hernández, P. (2004). Test Autoevaluativo Multifactorial de Adaptación Infantil (TAMAl). TEA Ediciones, S.A., Madrid (5a edición, revisada).

Hidalgo, R. y Davidson, J. (2000). Posttraumatic stress disorder: Epidemiology and health related considerations. Journal of Clinical Psychiatry, 61 (7), 5-13.
Horowitz, M., Wilner, N., \& Alvarez, W. (1979). Impact of Events Scale: a measure of subjective stress. Psychosomatic Medicine, 41, 209-218.

Jenkins, E.J. y Bell, C.C. (1994). Violence among inner city high school students and posttraumatic stress disorder. En S. Friedman (ed.). Anxiety disorders in African Americans. New York: Springer.

Kessler, R.C., Borges, B. y Walters, E.E. (1999). Prevalence and risk factors of lifetime suicide attempts in the National Comorbidity Survey. Archives of General Psychiatry, 56, 617-626.

Kessler, R.C., Sonnega, A., Bromet, E., Hughes, M. y Nelson, C.B. (1995). Posttraumatic stress disorder. National Comorbidity Survey. Archives of General Psychiatry, 52, 1048-1060.

Kilpatrick, D.G., Acierno, R., Saunder, B.E., Resnick, H.S., Best, C.L, y Schnurr, P.P. (2000). Risk factors for adolescent substance abuse and dependence: Data from a national sample. Journal of Consulting and Clinical Psychology, 68 (1), 19-30.

Kilpatrick, D.G., Ruggeiro, K.J, Acierno, R., Saunder, B.E., Resnick, H.S. y Best, C.L, (2003). Violence and risk of PTSD, major depression, substance abuse/dependence and comorbidity: Results from the National Survey of Adolescent. Journal of Con-sulting and Clinical Psychology, 71, 692-700.

Kovacs, M. (2004). Inventario de Depresión Infantil $(C D I)$. TEA ediciones, S.A., Madrid.

Lerer, B., Bleich, A., Kotler, M. Garb, R., Hertzberg, M. y Levin, B. (1987). Posttraumatic stress disorder in Israeli combat veterans: Effect of phenelzine treatment. Archives of General Psychiatry, 44, 976-981.

Linehan, M.N., Tutdk, D.A., Heard, H.L. y Armstrong, H.E. (1994). Interpersonal outcome of cognitive behavioural treatment for chronically suicidal borderline patients. American Journal of Psychiatry, 125, 1771-1776.

Lipschitz, D.S., Winegar, R.K. Hartnick, E., Foote, B. y Southwick, S.M. (1999). Posttraumatic stress disorder in hospitalized adolescents: psychiatric comorbidity and clinical correlates. Journal of the American Academy of Child and Adolescent Psychiatry, 38 (4), 385-393.

Lisak, D., Koper, J. y Song, P. (1996). Factors in the cycle of violence: gender rigidity and emotional constriction. Journal of Trauma Stress, 9, 721743.

López Soler, C., García Montalvo, C., Murcia, L., Martín, C., Cortegano, C., López Mora I y López García, G. (1995). Problemas psicopatológicos en una muestra clínica de niños-niñas: taxonomías empíricas. Anales de Psicología, 11(2), 129-142.

López Soler, C. (1999). Aportaciones del modelo de taxonomías empíricas al diagnóstico psicopatológico en infancia y ado-lescencia. Comunicación. II Con-greso de la Asociación española 
de Psicología Clínica y Psic-opatología. Libro de Resúmenes: 80-81. Murcia.

López Soler, C. (2006). Escala de situaciones traumáticas intrafamiliares. Universidad de Murcia. Documento no publicado.

López-Soler, C. (2008). Las reacciones postraumáticas en infancia y adolescencia maltratada: el trauma complejo. Revista de Psicopatología y Psicología Clínica, 13(3).

Luxenberg, T., Spinazzola, J. y van der Kolk, B. (2001). Complex Trauma and Disorders of Extreme Stress (DESNOS) Diagnosis, Part One: Assessment. Directions in Psychiatry, 21, lesson 25, 373-392.

March J., Amaya L., Pynoos R. (1997). Pediatric posttraumatic stress disorder. En: Wiener J. (ed). Text book of Child and Adolescent Psychiatry. 2nd ed. Washington DC, American Psychiatric Press, 507- 524.

Margolin, G. y Gordis, E.B. (2000). The effects of family and community violence on children. Annual Review of Psychology, 51, 445-479.

Márquez, M. (2000). Los trastornos por estrés: del trauma a la disociación. En: Grau, A. y Meneghello, J. (eds). Psiquiatría y Psicología de la Infancia y Adolescencia. Buenos Aires, Editorial Médica Panamericana, 369- 379.

Martínez, A., López-Soler, C., García Montalvo, C., Garriga, A., Alcántara, M.V. y Prieto, M. (2006). Entrevista Semiestructurada de maltrato infantil y adolescente. Universidad de Murcia. Documento no publicado.

McCloskey, L. (2000). Posttraumatic stress in children exposed to family violence and single event trauma. Journal of the American Academy of Child and Adolescent Psychiatry, 39, 108-115.

McFarlane, A. (1988). The phenomenology of posttraumatic stress disorders following a natural disaster. Journal of Nervous and Mental Disease, 176, 22-29.

Milgram N. (1993). Los niños y el estrés. En: Ollendick T, Hersen M (eds). Psicopatología Infantil. Barcelona, Martínez Roca, 464-483.

Montt, M. E. y Hermosilla, W. (2001). Trastorno de estrés post-traumático en niños. Revista Chilena de Neuro-psiquiatría, 39 (2), 110-120.

Moran, M. (2007). Developmental Trauma Merits DSM Diagnosis, Experts Say. Psychiatry News February 2, 42 (3), 20.

Muris, P. (1997). The Screen for Child Anxiety Related Emotional Disorders (revised version). Maastricht: Maastrict University, Department of Psychology.

Muris, P. et al (1999). The revised version of the screen for child anxiety related emotional disorders (SCARED-R): Further evidence for its reliability and validity. J. Anxiety, stress, and coping, 12, (4), 411-425

National Center for PTSD. Fact Sheet and Child measures (2006). http://www.ncptsd.vagov/fact/ specific/fs_children.html
Neumann, D.A., Houskamp, B.M., Pollock, V.E. y Briere, J. (1996). The long-term sequelae of childhood sexual abuse in women: A meta-analytic review. Child Maltreatment, 1, 6-16.

Norris, F.H. (1992). Epidemiology of trauma: frequency and impact of different potentially traumatic events on different demographic groups. Journal of Consulting \& Clinical Psychology, 60, 409-418.

Norris, F.H., Foster, J.D., \& Weisshaar, D.L. (2002). The epidemiology of sex differences in PTSD. A cross developmental, societal and research contexts. In R. Kimberling, P. Ouminette, \& J. Wolfe (Eds.). Gender and PTSD (pp. 3- 42). New York: Guilford Press.

Ohan, J.L., Myers, K., \& Collett, B.R. (2002). Tenyear review of rating scales IV: scales assessing trauma and its effects. Journal of American Academy of Child and Adolescent Psychiatry, 41, 1401-1422.

Palacios, L. y Heinze, G. (2002). Trastorno por Estrés Postraumático: una revisión del tema (primera parte). Salud mental, 25 (3), 19-26.

Pelcovitz, D., van der Kolk, B.A., Roth, S., Mandel, F, Kaplan, S. y Resick, P. (1997). Development of a criteria set and a structured interview for disorder of extreme stress (SIDES). Journal of Trauma Stress, 10, 3-16.

Perrin, S., Smith, P., \& Yule, W. (2000). Practitioner review: The assessment and treatment of posttraumatic stress disorder in children and adolescents. Journal of Child Psychology and Psychiatry and Allied Disciplines, 41, 277-289.

Pfefferbaum B. (1997). Posttraumatic Stress Disorder in children: a review of the past 10 years. Journal of American Academy of Child and Adolescent Psychiatry, 36, 1503-1511.

Pitman, R.K., Orr, S.P., Forgue, D.F., de Jong, J. y Claiborn, J.M. (1987). Psychophysiologic assessment of posttraumatic stress disorder imagery in Vietnam combat veterans. Archives of General Psychiatry, 44, 970-975.

Putnam, F. y Trickett, P.K. (1997). The psychobiological effects of sexual abuse, a longitudinal study. Annals of the New York Academy of Science, 821, 150-159.

Roth, S., Newman, E., Pelcovitz, D., van der Kolk, B.A. y Mandel, F. (1997). Complex PTSD in victims exposed to sexual and physical abuse: Results from the DSM-IV field trial for posttraumatic stress disorder. Journal of Trauma Stress, 10, 539-555.

Saigh, P.A. (2004). Assessment of PTSD in children and adolescents. En R. Silva (ed.). Posttraumatic stress disorders in children and adolescents. Handbook. New York: Norton.

Sandín, B. y Chorot, P. (1997). Versión española del Indice de Sensibilidad a la Ansiedad (CASI). En B. Sandín, Ansiedad, Miedos y Fobias en niños $y$ adolescentes. Madrid: Dykinson. 
Sandín, B., Chorot, P., Lostao, L, Joiner, T.E., Santed, M.A. y Valiente, R.M. (1999a). Escalas PANAS de afecto positive y negative. Validación factorial y convergencia transcultural. Psicothema, 14, 333-339.

Sarmiento, P., Fernández Gutiérrez, M. y Barbacil, A. (2006). Perspectivas actuales en el tratamiento del trastorno de estrés postraumático en la población infantil: Análisis bibliométrico. Clínica y Salud, 17 (1), 69-89.

Sauter, J. y Franklin, C. (1998). Assessing posttraumatic stress disorder in children: diagnostic and measurement strategies. Research on Social Work Practice, 8, 251-271.

Saylor, C.F., Cupit, C., Stokes, S. y Taylor, M. (1999). The Pediatric Emotional Distress Scale: a brief screening measure for young children exposed to traumatic events. Journal of Clinical Child Psychology, 28.

Scheeringa, M.S., Zeanah, C.H., Drell, M.J. y Larrieu, J. (1995). Two approaches to the diagnosis of posttraumatic stress disorder in infancy and early childhood. Journal of the American Academy of Child and Adolescent Psychiatry, 34 (2), 191-200.

Scheeringa, M.S., Zeanah, C.H., Meyeres, L. y Putnam, F.W. (2003). New findings on alternative criteria for PTSD in preschool children. Journal of the American Academy of Child and Adolescent Psychiatry, 42 (5), 561-570.

Shalev, A.Y. y Rogel-Fuchs, Y. (1993). Psychophysiology of PTSD: From sulphur fumes to behavioural genetics. Journal of Nervous Mental Disease, 55, 413-423.

Shannon, M., Lunigan, C., Finch, A. y Taylor, C. (1994). Children exposed to disaster: I. Epidemiology of post-traumatic symptoms and symptom profiles. Journal of American Academy of Child and Adolescent Psychiatry, 33, 80-93.

Shore, J.H., Vollmer, W.M. y Tatum, E.I. (1989). Community patterns of posttraumatic stress disorders. Journal of Nervous and Mental Disease, 177, 681-685.

Sierles, F.S., Chen, J., McFarland, R.E. y Taylor, M.A. (1983). Posttraumatic stress disorder and concurrent psychiatric illness: a preliminary report. American Journal of Psychiatry, 140, 1177-1179.

Silva, F. y Martorell, M.C. (2001). Batería de Socialización. Autoevaluación (BAS-3). TEA Ediciones, S.A., Madrid. (3 $3^{a}$ edición).

Spielberger, C. D. (1973). Inventario de Ansiedad Estado - Rasgo para niños, STAIC. Palo Alto, CA. Consulting Psychologists Press.

Spielberger, C. D., Goursch, R. L. \& Lushene, R. E. (1982). Cuestionario de Ansiedad Estado - Rasgo, STAI. Madrid. TEA Ediciones

Spinazzola, J., Blaustein, M., Kisiel, C. y van der Kolk, B. (2001). Beyond PTSD: Further evidence for a complex adaptational response to traumatic life events. Paper presented at the American Psychiatric Association Annual Meeting, New Orleans.

Stuber, M., Nader, K., Yasuda, P., Pynoos, R. y Cohen, S. (1991). Stress responses alter pediatric home marrow transplantation: Preliminary results of a prospective longitudinal study. Journal of the American Academy of Child and Ado-lescent Psychiatry, 30, 952-957.

Summit, R. (1983). The child sexual abuse accommodation syn-drome. Child Abuse \& Neglect, 7 (2), 177-193.

Terr L.C. (1983). Chowchilla revisited: the effects of psychic trauma four years after a schoolbus kidnapping. American Journal of Psychiatry 140, 1543-1550.

Terr, L. (1985). Psychic trauma in children and adolescents. Psychiatric Clinical of North America, 8, 815-835.

Terr, L.C. (1979). Children of Chowchilla: a study of psychic trauma. The Psychoanalytic Study of the Child, 34, 552-623.

Terr, L.C. (1981). Psychic trauma in children: observations following the Chochilla school-bus kidna-pping. American Journal of Psychiatry, 38, 14-19.

Tierney, J.A. (2000). Post-traumatic stress disorder in children: controversies and unresolved issues. Journal of Child and Adolescent Psychiatric Nursing, 13, 147-160.

Valdivia, M. (2002). Trastorno por estrés postraumático en la niñez. Revista Chilena de NeuroPsiquiatría, 40 (2), 76-85.

Van der Kolk, B. (2001). The Assessment and Treatment of Complex PTSD. In Rachel Yehuda (ed.). Traumatic Stress. American Psychiatric Press.

Van der Kolk, B. (2005). Child Abuse \& Victimization. Psychiatric Annals, 374-378

Van der Kolk, B., Roth, S., Pelcovitz, D., Sunday, S. y Spinazzola, J. (2005). Disorders of Extreme Stress: The Empirical Foundation of a Complex Adaptation to Trauma. Journal of Traumatic Stress, 18 (5), 389-39

Van der Kolk, B.A. (1985). Adolescent vulnerability to posttraumatic stress. Psychiatry, 48, 365-370.

Van der Kolk, B.A. (1996). The complexity of adaptation to trauma: Self-regulation, stimulus discrimination, and characterological development. In: van der Kolk B.A., McFarlane A., Weisaeth L. (eds.) Traumatic stress: The Effects of Overwhelming Experience on Mind, body, and Society. New York: Guilford Press, 182-213.

Van der Kolk, B.A., Pelcovitz, D., Roth, S.H., Mandel, F., McFarlane, A., y Herman, J.L. (1996). Dissociation, somatization, and affect dysregulation: The com-plexity of adaptation to trauma. American Journal of Psychiatry, 153, 83-93.

Van der Kolk, B.A., Perry, J.C. y Herman, J.L. (1991). Childhood origins of self-destructive behaviour. American Journal of Psychiatry, 148, 1665- 
1671.

Van Kampen, M., Watson, C.G. y Tilleskjor, C. (1986). The defi-nition of posttraumatic stress disorder in alcoholic Vietnam ve-terans. Are the DSM-III diagnostic criteria necessary and su-fficient?. Journal of Nervous and Mental Disease, 174, 137-144.

Wagnild, G. y Young, H.M. (1993). Development and psychometrics evaluation of the Resilience Scale. Journal of Nursing Measurement, 1, 165-177.

Walker, L. (1984). The battered woman syndrome. USA, Spring Publishing Company.

Wang, C.T. y y Daro, D. (1997). Current trends in child abuse reporting and fatalities: The results of the 1997 annual fifty state survey. Chicago: National Committee to Prevent Child Abuse.

Wilson, S.N., van der Kolk, B.A., Burbridge, J.A., Filser, R.E. y Kradin, R. (1999). Pshenotype of blood lymphocytes in PTSD suggests chronic immune acti-vation. Psychosomatics, 40, 222-225.

Wolfe, V.V., Gentile, C., Michienzi, T., Sas, L., \& Wolfe, D. (1991). The Childrens's Impact of Traumatic Events Scale: A measure of post-sexual-abuse PTSD sym-ptoms. Behavioral Assessment, 13, 359-383.

Yehuda, R. (1999). Linking the neuroendocrinology of post-traumatic stress disorder with recent neuroanatomic findings. Seminars in Clinical
Neuro-psychiatry, 4, 256-265

Zlotnick, C. y Pearlstein, T. (1997). Validation of the structured interview for Disorders of Extreme Stress. Comprehensive Psychiatry, 38, 243-247.

Zlotnitk, C., Zakriski, A.L., Shea, M.T., Costello, E., Begin, A., Pearlstein, T. y Simpson, E. (1996). The long-term sequelae of sexual abuse: Support for a complex posttraumatic stress disorder. Journal of Traumatic Stress, 9 (2), 195-205. 